\section{Putting the brakes on T cell hyperactivity in SLE}

The RNA-binding protein serine/ arginine-rich splicing factor 1 (SRSF1) restrains T cell hyperactivity and systemic autoimmunity in systemic lupus erythematosus (SLE), according to a new study. The researchers found that in $\mathrm{T}$ cells, SRSF1 suppresses the activity of the mechanistic target of rapamycin (mTOR) pathway by promoting the expression of PTEN (a negative regulator of the mTOR pathway), but that this braking system is potentially dysregulated in SLE.

"Patients with SLE and other autoimmune diseases have an overactive immune response," explains corresponding author Vaishali Moulton. "Defects in signalling and gene regulation in T cells from patients with SLE are linked to the hyperactive phenotype of these cells, which makes them produce high amounts of aberrant inflammatory cytokines."

Previous findings had implicated SRSF1 in T cell dysfunction in SLE.
To investigate this link further, the researchers generated mice with T cell-specific deletion of Srsf 1 . The mice developed systemic autoimmunity, as indicated by the presence of circulating SLE-associated autoantibodies and kidney disease resembling lupus nephritis.

Notably, the T cells from these mice had a hyperactive phenotype and produced pro-inflammatory cytokines (including IL-17, IL-4 and IFN $\gamma$ ). Furthermore, the activity of mTOR was increased in these cells, whereas the expression of PTEN was downregulated. Using a luciferase report assay, the researchers found

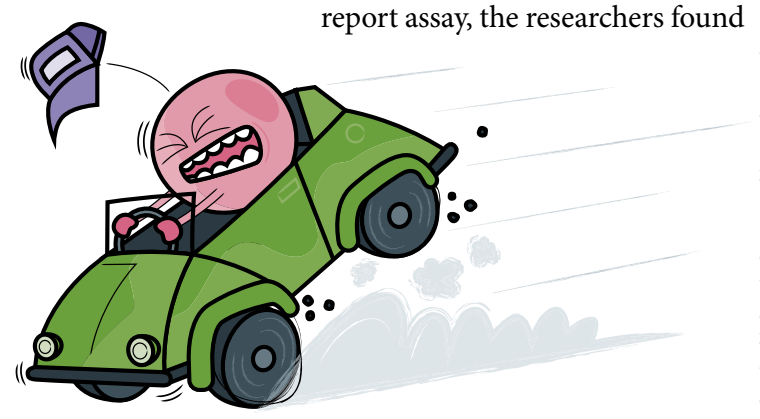

that SRSF1 post-transcriptionally controlled the expression of PTEN.

Treatment with rapamycin, an mTOR pathway inhibitor, reduced the production of pro-inflammatory cytokines by SRSF1-deficient T cells in vitro. Furthermore, rapamycin treatment reduced serum autoantibodies titres and inhibited $T$ cell infiltration into the kidneys in SRSF1-deficient mice.

Interestingly, $\mathrm{T}$ cells from patients with SLE expressed low levels of PTEN that correlated with low levels of SRSF1. Transient transfection of $\mathrm{T}$ cells from patients with SLE with an SRSF1-expressing vector increased the expression of PTEN, reduced the activity of mTOR and decreased the production of IL-17 and IFN $\gamma$.

"These findings open up an exciting unexplored avenue for future studies and could pave the path for new molecules and pathways as targets for therapy or biomarkers," concludes Moulton.

Jessica McHugh

ORIGINAL ARTICLE Katsuyama, T. et al. Splicing factor SRSF1 controls T cell hyperactivity and systemic autoimmunity.J. Clin. Invest. https://doi. org/10.1172/JCl127949 (2019)

\section{GOUT}

\section{MSU crystals at-TAK!}

In gout, monosodium urate (MSU) crystals trigger IL- $1 \beta$ production, but exactly how MSU crystals mediate this process is not well understood. A new study suggests that MSU crystals can stimulate inflammation by interacting with transforming growth factor- $\beta$ activated kinase 1 (TAK1; also known as MAP3K7), which regulates the expression of several pro-inflammatory cytokines including IL-1 $\beta$ and TNF.

"Studies so far have emphasized the primary role of the NLRP3 inflammasome in MSU crystal-induced inflammation," explains corresponding author Salah Ahmed. "This is the first study to identify the molecular and post-translational mechanisms triggered by MSU crystals in macrophages and to propose TAK 1 as a potential therapeutic target for gout."

In vitro studies in THP-1 macrophages and human monocyte-derived macrophages revealed that MSU crystals could activate proteins involved downstream in the IL- $1 \beta$ signalling pathway (including TAK1)

4 urate molecules can directly bind to TAK 1 and arrest the kinase in an activated state without the need for IL-1 $\beta$ itself. Further in vitro investigations showed that MSU crystals also modulated protein ubiquitination and the expression of deubiquitination enzymes, thereby accelerating proinflammatory cytokine production. In THP-1 macrophages, administration of the TAK1 inhibitor 5Z-7-oxozeaenol (5ZO) suppressed MSU crystal-induced production of pro-inflammatory cytokines, including IL-1 $\beta$. Molecular dynamics simulations revealed that urate molecules can directly bind to TAK 1 and arrest the kinase in an activated state, potentially explaining the effects of MSU crystals on TAK1.

In mice with MSU crystal-induced inflammation (a model for gout), administration of $5 Z O$ prior to disease onset inhibited inflammation and reduced disease severity to a level comparable with that induced by the urate-lowering therapy febuxostat.

"Our plan is to extend these findings into macrophages from patients with gout to further validate the mechanism in a clinical population," says Ahmed. "These findings might also be applied in other autoimmune diseases where IL-1 $\beta$ has a central role," he suggests.

Joanna Collison

ORIGINAL ARTICLE Singh, A. K. et al.

Suppression of monosodium urate crystal-induced inflammation by inhibiting TGF- $\beta$-activated kinase 1-dependent signaling: role of the ubiquitin proteasome system. Cell. Mol. Immunol. https:// doi.org/10.1038/s41423-019-0284-3 (2019) 